\title{
Vector Meson Propagator and Baryon Current Conservation
}

\author{
S. S. Wu, H. X. Zhang, Y. J. Yao \\ Center for Theoretical Physics and Department of Physics \\ Jilin University, Changchun 130023 \\ People's Republic of China
}

\begin{abstract}
If baryons couple only with $\omega$-mesons, one found the baryon spectral function may be negative. We show this unacceptable result is caused by the $k_{\mu} k_{\nu}$-terms in the $\omega$-meson propagator. Their contribution may not vanish in approximate calculations which violate the baryon current conservation. A rule is suggested, by which the calculated baryon spectral function is well behaved.
\end{abstract}

PACS: 21.60. $\mathrm{J}_{z}, 21.65+\mathrm{f}, 11.10 \mathrm{Gh}$

Keywords: Vector meson propagator, baryon current conservation

Krein, Nielsen, Puff and Wilets (KNPW) [1] pointed out not long ago that in a self-consistent (SC) Hartree-Fock (HF) calculation of the renormalized baryon propagator for the case of zero-density the spectral function $A_{R}(\kappa)$ can be negative for some real values of $\kappa$, if baryons couple only with $\omega$-mesons. They emphasized that this is unacceptable. The spectral representation they considered is of the form

$$
G(k)=-\int_{-\infty}^{\infty} d \kappa A_{R}(\kappa) \frac{\gamma_{\mu} k_{\mu}+i \kappa}{k^{2}+\kappa^{2}-i \epsilon}
$$

Since $A_{R}(\kappa)$ represents the probability that a state of mass $|\kappa|$ is created, it must be non-negative. They suggested that it might be due to the inadequacy of the HF approximation or the inconsistency of the theory. In their calculation they have neglected all the terms proportional to $k_{\mu} k_{\nu}$ in the $\omega$ meson propagator on the basis of the baryon current conservation implied by the model for a rigorous calculation. Though this is a generally accepted approximation [2] and indeed, such terms need not be taken into account if the baryon current conserves, their contribution in the SCHF approximation 
is not zero and has to be studied. It also indicates that the SCHF approximation does not preserve the baryon current conservation. The aim of this letter is to show that the baryon spectral function becomes non-negative and is well behaved if an adequate part of the $k_{\mu} k_{\nu}$-terms is included.

For a system of baryons coupling only with $\omega$-mesons, the Lagrangian density has the form

$$
L=-\bar{\psi}\left(\gamma_{\mu} \partial_{\mu}+M\right) \psi-\frac{1}{4} F_{\mu \nu} F_{\mu \nu}-\frac{1}{2} m_{v}^{2} A_{\mu} A_{\mu}+i g_{v} \bar{\psi} \gamma_{\mu} \psi A_{\mu}+L_{C T C}
$$

where $F_{\mu \nu}=\partial_{\mu} A_{\nu}-\partial_{\nu} A_{\mu}, \partial_{\mu} \equiv \partial / \partial x_{\mu}, x_{\mu}=\left(\vec{x}, i x_{0}\right), x^{2}=x_{\mu} x_{\mu}=\vec{x}^{2}-$ $x_{0}^{2}$ with $x_{0} \equiv t$ and CTC means the counterterm correction introduced for the purpose of renormalization. The baryon and $\omega$-meson propagators are defined as

$$
\begin{gathered}
G_{\alpha \beta}\left(x=x_{1}-x_{2}\right)=\left\langle T\left[\psi_{\alpha}\left(x_{1}\right) \bar{\psi}_{\beta}\left(x_{2}\right)\right]\right\rangle=\int \frac{d^{4} k}{(2 \pi)^{4}} e^{i k_{\rho} x_{\rho}} G_{\alpha \beta}(k), \\
D_{\mu \nu}(x)=\left\langle T\left[A_{\mu}\left(x_{1}\right) A_{\nu}\left(x_{2}\right)\right]\right\rangle=\int \frac{d^{4} k}{(2 \pi)^{4}} e^{i k_{\rho} x_{\rho}} D_{\mu \nu}(k),
\end{gathered}
$$

where $\langle 0\rangle \equiv\left\langle\Psi_{0}|0| \Psi_{0}\right\rangle$ and $\left|\Psi_{0}\right\rangle$ denotes the exact ground state. The dressed HF scheme is illustrated in Fig. 1, where $\bar{G}(\bar{D})$ denotes an appropriate expression chosen for the calculation of the baryon ( $\omega$-meson) propagator in the self-energy. As is wellknown, the relevant Dyson-Schwinger (DS) equations can be written as follows:

(a) for baryon

$$
\begin{gathered}
G(k)=G^{0}(k)+G^{0}(k) \Sigma(k) G(k)=-\left[\gamma_{\mu} k_{\mu}-i M+\Sigma(k)\right]^{-1}, \\
\Sigma(k)=g_{v}^{2} \int \frac{d^{\tau} q}{(2 \pi)^{4}} \Gamma_{\eta}(k, q, k-q) \bar{G}(q) \bar{D}_{\eta \lambda}(k-q) \gamma_{\lambda}+\Sigma_{C T C}(k) ;
\end{gathered}
$$

(b) for $\omega$-meson

$$
\begin{gathered}
D_{\mu \nu}(k)=D_{\mu \nu}^{0}(k)+D_{\mu \eta}^{0}(k) \Pi_{\eta \lambda}(k) D_{\lambda \nu}(k), \\
\widehat{\Pi}_{\eta \lambda}(k)=-g_{v}^{2} \int \frac{d^{\tau} q}{(2 \pi)^{4}} \operatorname{Tr}\left[\Gamma_{\eta}(k+q, k, q) \bar{G}(k+q) \gamma_{\lambda} \bar{G}(q)\right],
\end{gathered}
$$

where a caret indicates that the quantity is not yet renormalized, $\tau=4-$ $\delta\left(\delta \rightarrow 0^{+}\right)$and in Eq. (5) the Feynman prescription $M \rightarrow M-i \epsilon$ is understood. Since the baryon current is conserved, we have

$$
\widehat{\Pi}_{\eta \lambda}(k)=\left(\delta_{\eta \lambda}-\frac{k_{\eta} k_{\lambda}}{k^{2}}\right) \widehat{\Pi}_{v}(k)
$$


which gives $\widehat{\Pi}_{v}(k)=\frac{1}{3} \sum_{\eta} \widehat{\Pi}_{\eta \eta}(k)$. Eq. (9) shows that the renormalized $\Pi_{\eta \lambda}$ can be obtained from

$$
\Pi_{v}(k)=\widehat{\Pi}_{v}(k)+\Pi_{C T C}(k)
$$

The renormalized counterterms will be fixed by the following conditions

$$
\begin{array}{cc}
\left.\Sigma(k)\right|_{\gamma_{\mu} k_{\mu}=i M_{t}}=0 ; & \left.\frac{\partial \Sigma(k)}{\partial\left(\gamma_{\mu} k_{\mu}\right)}\right|_{\gamma_{\mu} k_{\mu}=i M_{t}}=0, \\
\left.\Pi_{v}(k)\right|_{k^{2}=0}=0 ; & \left.\frac{\partial \prod_{v}(k)}{\partial k^{2}}\right|_{k^{2}=0}=0,
\end{array}
$$

where $M_{t}$ is the true baryon mass. Following [3], we shall use the following notations to distinguish different approximations: scheme P, $\bar{G}=-\left[\gamma_{\mu} k_{\mu}-\right.$ $\left.i M_{t}\right]^{-1}, \bar{D}_{\eta \lambda}=D_{\eta \lambda}^{0}$; scheme BP, $\bar{G}=G, \bar{D}_{\eta \lambda}=D_{\eta \lambda}^{0}$ and scheme FSC, $\bar{G}=G, \bar{D}_{\eta \lambda}=D_{\eta \lambda}$. It is seen that in scheme BP Eq. (5) has to be solved self-consistently $[1,4-6]$, while in scheme FSC [7, 3] one should consider a selfconsistent coupled set of renormalized DS equations (5-10). As emphasized in [7], the study of an ansatz consistent with the Ward-Takahashi identity may provide a way to preserve the baryon current conservation. Though it does not hold in the SCHF approximation, we shall assume that Eq. (9) is valid approximately. We note that $D_{\mu \nu}$ can be written as $[8,9]$

$$
\begin{gathered}
D_{\mu \nu}(k)=\left(\delta_{\mu \nu}-\frac{k_{\mu} k_{\nu}}{k^{2}}\right) \Delta_{v}(k)-i \frac{k_{\mu} k_{\nu}}{k^{2}\left(m_{v}^{2}+\delta m_{v}^{2}\right)} \\
\Delta_{v}(k)=\Delta_{v}^{0}(k)+\Delta_{v}^{0}(k) \Pi_{v}(k) \Delta_{v}(k)=-i\left[k^{2}+m_{v}^{2}+i \Pi_{v}(k)-i \epsilon\right]^{-1}
\end{gathered}
$$

where $\delta m_{v}^{2}$ is the mass counterterm for the $\omega$-meson. As is wellknown [10], a properly normalized spectral representation for the baryon propagator equivalent to Eq. (1) can be written in the form:

$$
\widetilde{G}(k)=-Z_{2} \frac{\gamma_{\mu} k_{\mu}+i M_{t}}{k^{2}+M_{t}^{2}-i \epsilon}-\int_{m_{1}^{2}}^{\infty} d \sigma^{2} \frac{\gamma_{\mu} k_{\mu} \alpha\left(-\sigma^{2}, Z_{2}\right)+i M_{t} \beta\left(-\sigma^{2}, Z_{2}\right)}{k^{2}+\sigma^{2}-i \epsilon},
$$

where $m_{1}=M_{t}+m_{v}$ is the threshold of the continuum spectrum,

$$
Z_{2}+\int_{m_{1}^{2}}^{\infty} d \sigma^{2} \alpha\left(-\sigma^{2}, Z_{2}\right)=1
$$

and the spectral functions $\alpha$ and $\beta$ should have the following properties: 


$$
\begin{aligned}
& \text { (a). they are all real, } \\
& \text { (b). } \alpha\left(-\sigma^{2}, Z_{2}\right) \geq 0, \\
& \text { (c). } \sigma \alpha\left(-\sigma^{2}, Z_{2}\right)-M_{t} \beta\left(-\sigma^{2}, Z_{2}\right) \geq 0 \text {. }
\end{aligned}
$$

For the $\omega$-meson propagator we have

$$
\begin{gathered}
\widetilde{\Delta}_{v}(k)=Z_{v} \frac{-i}{k^{2}+\widehat{m}_{v}^{2}-i \epsilon}-i \int_{t h}^{\infty} d m^{2} \frac{\rho_{v}\left(-m^{2}, Z_{v}\right)}{k^{2}+m^{2}-i \epsilon}, \\
Z_{v}+\int_{t h}^{\infty} d m^{2} \rho_{v}\left(-m^{2}, Z_{v}\right)=1 .
\end{gathered}
$$

In Eq. (17) $\widehat{m}_{v}$ denotes the true $\omega$-meson mass and $t h=\left(2 M_{t}\right)^{2}$ the threshold of the continuum. Since $G(k)$ and $\widetilde{G}(k)$ as well as $\Delta_{v}(k)$ and $\widetilde{\Delta}_{v}(k)$ are normalized differently, we should write $\widetilde{G}(k)=Z G(k)$ and $\widetilde{\Delta}_{v}(k)=Y_{v} \Delta_{v}(k)$. It is easily seen that $Z_{2}=Z Z_{t}$ and $Z_{v}=Y_{v} R_{v}$, where $\left(-Z_{t}\right)$ and $\left(-i R_{v}\right)$ are the residues of $G(k)$ and $\Delta_{v}(k)$ at the poles $\gamma_{\mu} k_{\mu}=i M_{t}$ and $k^{2}=-\widehat{m}_{v}^{2}$, respectively. In the case of zero-density one may write

$$
\Sigma(k)=\gamma_{\mu} k_{\mu} a\left(k^{2}\right)-i M b\left(k^{2}\right) .
$$

We note that under the on-shell condition (11a) one has $Z_{t}=1$ and $M=M_{t}$ [6]. Neglecting all the $k_{\mu} k_{\nu}$ terms in Eq. (12) and substituting $\bar{D}_{\mu \nu}=$ $\delta_{\mu \nu} \Delta_{v}=\delta_{\mu \nu} Y_{v}^{-1} \widetilde{\Delta}_{v}$ as well as $\bar{G}=Z^{-1} \widetilde{G}$ in Eqs. (6) and (8), an explicit coupled set of renormalized equations for the determination of $(a, b),(\alpha, \beta)$, $\Pi_{v}$ and $\rho_{v}$ has been given in [3]. Since the expressions for the rest of the equations considered here are the same as in [3], for space saving and for convenience of discussion only a part of them will be written down as follows:

$$
\begin{gathered}
a\left(k^{2}\right)=a_{\delta}\left(k^{2}\right)+a_{\Delta}\left(k^{2}\right) ; \quad b\left(k^{2}\right)=b_{\delta}\left(k^{2}\right)+b_{\Delta}\left(k^{2}\right) \\
a_{\delta}\left(k^{2}\right)=\frac{g_{v}^{2}}{8 \pi^{2}} \int_{0}^{\infty} \int_{0} d \sigma^{2} d m^{2} \int_{0}^{1} d x f_{\alpha}\left(-\sigma^{2}\right) h_{v}\left(-m^{2}\right)(1-x) \ln \frac{K^{2}\left(-M_{t}^{2}\right)}{K^{2}\left(k^{2}\right)}+c_{\delta} \\
b_{\delta}\left(k^{2}\right)=\frac{g_{v}^{2}}{4 \pi^{2}} \iint_{0}^{\infty} d \sigma^{2} d m^{2} \int_{0}^{1} d x f_{\beta}\left(-\sigma^{2}\right) h_{v}\left(-m^{2}\right) \ln \frac{K^{2}\left(-M_{t}^{2}\right)}{K^{2}\left(k^{2}\right)}+c_{\delta} \\
f_{\gamma}\left(-\sigma^{2}\right)=\delta\left(\sigma^{2}-M_{t}^{2}\right)+\theta\left(\sigma^{2}-m_{1}^{2}\right) \gamma\left(-\sigma^{2}\right), \quad(\gamma=\alpha \text { or } \beta) \\
h_{v}\left(-m^{2}\right)=R_{v} \delta\left(m^{2}-\widehat{m}_{v}^{2}\right)+\theta\left(m^{2}-t h\right) \rho_{v}\left(-m^{2}\right) \\
K^{2}\left(k^{2}\right) \equiv K^{2}\left(x, k^{2}, \sigma^{2}, m^{2}\right)=x(1-x) k^{2}+x \sigma^{2}+(1-x) m^{2}
\end{gathered}
$$


where $\gamma\left(-\sigma^{2}\right)=Z^{-1} \gamma\left(-\sigma^{2}, Z_{2}\right)$ and $\rho_{v}\left(-m^{2}\right)=Y_{v}^{-1} \rho_{v}\left(-m^{2}, Z_{v}\right)$. In addition, we have

$$
\begin{gathered}
\alpha\left(k^{2}\right)=\frac{1}{\pi} \operatorname{Im} \frac{1+a\left(k^{2}\right)}{D\left(k^{2}\right)} ; \quad \beta\left(k^{2}\right)=\frac{1}{\pi} \operatorname{Im} \frac{1+b\left(k^{2}\right)}{D\left(k^{2}\right)}, \\
D\left(k^{2}\right)=\left[1+a\left(k^{2}\right)\right]^{2} k^{2}+\left[1+b\left(k^{2}\right)\right]^{2} M_{t}^{2} .
\end{gathered}
$$

In Eq. (19) the $\Delta$-terms are contributed by the second $k_{\mu} k_{\nu}$-term in the round brackets of Eq. (12). They are given by

$$
\begin{gathered}
a_{\Delta}\left(k^{2}\right)=-\frac{g_{v}^{2}}{16 \pi^{2}} \iint_{0}^{\infty} d \sigma^{2} d m^{2} \int_{0}^{1} d x \int_{0}^{1-x} d y f_{\alpha}\left(-\sigma^{2}\right) h_{v}\left(-m^{2}\right) \times \\
\left\{(1+3 x) \ln \frac{L^{2}\left(-M_{t}^{2}\right)}{L^{2}\left(k^{2}\right)}-x^{2}(1-x)\left[\frac{k^{2}}{L^{2}\left(k^{2}\right)}+\frac{M_{t}^{2}}{L^{2}\left(-M_{t}^{2}\right)}\right]\right\}+c_{\Delta} \\
b_{\Delta}\left(k^{2}\right)=-\frac{g_{v}^{2}}{16 \pi^{2}} \iint_{0}^{\infty} d \sigma^{2} d m^{2} \int_{0}^{1} d x \int_{0}^{1-x} d y f_{\beta}\left(-\sigma^{2}\right) h_{v}\left(-m^{2}\right) \times \\
\left\{2 \ln \frac{L^{2}\left(-M_{t}^{2}\right)}{L^{2}\left(k^{2}\right)}+x^{2}\left[\frac{k^{2}}{L^{2}\left(k^{2}\right)}+\frac{M_{t}^{2}}{L^{2}\left(-M_{t}^{2}\right)}\right]\right\}+c_{\Delta} \\
L^{2}\left(k^{2}\right) \equiv L^{2}\left(x, y, k^{2}, \sigma^{2}, m^{2}\right)=x(1-x) k^{2}+x \sigma^{2}+y m^{2},
\end{gathered}
$$

where in Eqs. (19) and (21) we have $c_{\kappa}=2 M_{t}^{2}\left[a_{\kappa}^{\prime}\left(-M_{t}^{2}\right)-b_{\kappa}^{\prime}\left(-M_{t}^{2}\right)\right], \kappa=\delta$ or $\Delta$, and $f^{\prime}\left(k^{2}\right)=d f\left(k^{2}\right) / d k^{2}$.

As shown in Eq. (20), in order to find $\alpha$ and $\beta$ we need to know the imaginary parts of $a$ and $b$. Eqs. (19) and (21) show that $a$ and $b$ will be complex if either $K^{2}\left(k^{2}\right)$ or $L^{2}\left(k^{2}\right)$ becomes negative. Define $\theta_{\kappa} \equiv \theta_{\kappa}\left(x, \sigma^{2}, m^{2}, k^{2}\right)=0$ if $K^{2}\left(k^{2}\right)>0$ and $\theta_{\kappa}=1$ if $K^{2}\left(k^{2}\right)<0$. We may write $\ln K^{2}\left(k^{2}\right)=\ln \left|K^{2}\left(k^{2}\right)\right| \pm i \theta_{\kappa}(\pi+2 n \pi)$. Even if we set $n=0$, $\ln K^{2}$ is still indeterminate in case $\theta_{\kappa}=1$. Let the sign before $i \pi$ be denoted by $\sin K^{2}$. Obviously the same remark also applies to $\ln L^{2}\left(k^{2}\right)$. We shall make the stipulation that $\sin K^{2}$ and $\sin L^{2}$ are to be so chosen that Eq. (16) should be satisfied. Since $a$ and $b$ are derived from the same righthand side of Eq. (6), their $\sin K^{2}\left(\sin L^{2}\right)$ should be the same. According to [3], the above coupled set of equations can be solved easily by the method of iteration. We have solved it for schemes BP and FSC. In the following we shall choose $M_{t}=4.7585 \mathrm{fm}^{-1}, \widehat{m}_{v} / M_{t}=0.83387$ and $\bar{g}_{v}^{2}=g_{v}^{2} / 8 \pi^{2}=1.3685$. If we neglect all the $k_{\mu} k_{\nu}$-term in Eq. (12), it is impossible to make $\alpha\left(k^{2}\right)$ non-negative by an appropriate choice of $\sin K^{2}$ and $\sin L^{2}$. This is unacceptable and confirms the result of KNPW. As is wellknown, the last term in Eq. (12) is not renormalizable and the model becomes renormalizable only, because the $\omega$-meson couples to the conserved baryon current so that this term may be neglected. Thus we have calculated the additional contribution of the second term in the round brackets of Eq. (12) according to Eq. (21). 
The numerical results for $\sin K^{2}=-$ and $\sin L^{2}=+$ are shown in Fig. 2a. They are the best results we can find and satisfy all the requirements of Eq. (16). However, they are still physically unacceptable, because the threshold is wrong. It should be $-\left(M_{t}+\widehat{m}_{v}\right)^{2}$ rather than $-M_{t}^{2}$. This implies that the results contain a contribution from an additional zero-mass meson, which does not exist in our model. Clearly the latter is caused by the fact that $\frac{k_{\mu} k_{\nu}}{k^{2}} \Delta_{v}$ contains an additional zero-mass pole, because $k^{2}=\vec{k}^{2}-k_{0}^{2}=0$ does not ensure that $k_{\mu}$ and $k_{\nu}$ are both zero. Since Eq. (12) is formally rigorous and $D_{\mu \nu}$ should contain no zero-mass pole, the renormalized contribution of the latter must be cancelled by a finite part arising from the last term. Indeed, this can also be seen from the zero-order approximation to $D_{\mu \nu}$, which reads

$$
D_{\mu \nu}^{0}(k)=\left(\delta_{\mu \nu}-\frac{k_{\mu} k_{\nu}}{k^{2}}\right) \Delta_{v}^{0}(k)-i \frac{k_{\mu} k_{\nu}}{k^{2} m_{v}^{2}}=\left(\delta_{\mu \nu}+\frac{k_{\mu} k_{\nu}}{m_{v}^{2}}\right) \Delta_{v}^{0}(k),
$$

where $\Delta_{v}^{0}(k)=-i\left[k^{2}+m_{v}^{2}-i \epsilon\right]^{-1}$. Thus, if we neglect the last term in Eq. (12), $D_{\mu \nu}^{0}$ will contain a false zero-mass pole. Eq. (22) indicates that this last term makes the pole structure of $D_{\mu \nu}^{0}$ correct, though unrenormalizable. Our task is then to eliminate the contribution of the zero-mass meson from Eq. (21). This can be done unambiguously. We note that the integrals over the parameter $y$ in Eq. (21) can be performed exactly and in an elementary way. The expressions are, however, too lengthy to write down here. Let the curved brackets in Eqs. (21a and b) be denoted by $Y_{c}(y)$ with $c=a$ and $b$, respectively. We may express the integration over $y$ as

$$
\int_{0}^{1-x} d y Y_{c}(y)=Z_{c}(1-x)-Z_{c}(0)
$$

Set $L^{2}(y) \equiv L^{2}\left(x, y, k^{2}, \sigma^{2}, m^{2}\right)$. Eq. (19e) and (21c) show

$$
L^{2}(y=0)=K^{2}\left(x, k^{2}, \sigma^{2}, 0\right) ; \quad L^{2}(y=1-x)=K^{2}\left(x, k^{2}, \sigma^{2}, m^{2}\right) .
$$

Form Eqs. (21), (23) and (24) one easily finds that the contributions to the wrong threshold come exclusively from $Z_{c}(0)$. Substituting Eq. (23) in Eq. (21), discarding the contribution of $Z_{c}(0)$ and using Eq. (19), we have again solved the coupled set of equations. Fig. $2 \mathrm{~b}$ depicts our numerical results for $\alpha\left(k^{2}\right)$ and $\beta\left(k^{2}\right)$ with the choice $\sin K^{2}=-$ and $\sin L^{2}=-$. They satisfy all the requirements of Eq. (16) and have a correct threshold. Thus they are now acceptable. As displayed in Fig. 2, the effect of self-consistency on the baryon spectral function is perceptible, though the results of schemes $\mathrm{BP}$ and FSC are almost the same. The latter asserts, in agreement with [3], that there is no need to include the meson propagator in the self-consistency 
requirement. Clearly the fulfillment of Eq. (16) also implies that $A_{R}(\kappa)$ will be non-negative. Our results show if in an approximation the contribution of the renormalizable $k_{\mu} k_{\nu}$-term in $D_{\mu \nu}$ is not zero, it must be taken account of, otherwise the result may be qualitatively wrong. According to our above results the rule for the calculation of this additional contribution may be stated as follows: for $c=a$ or $b, Z_{c}(0)$ and the last term in Eq. (12) should both be discarded, because the contributions of the former and of a finite part from the latter cancel each other, while for the sake of renormalization the infinite nonrenormalizable part need not be considered on the basis of baryon current conservation. The latter can be understood as follows. Let $A=A_{0}+A_{r}+A_{\infty}$ be a term calculated in the SCHF approximation, where the subscripts $0, r$ and $\infty$ denote the contributions from the first, second (renormalizable) and third (nonrenormalizable) term in Eq. (12), respectively. Since the model possesses the baryon current conservation, if $A$ violates it, there must exist another term $B$ in the theory such that $A+B$ preserves it. Thus, $A_{r}+B_{r}$ and $A_{\infty}+B_{\infty}$ can be neglected. Since the infinity is an indefinite quantity and it always contains an undefined finite part (indicated by $f$ ), we may write $A_{\infty}+B_{\infty}=A_{\infty}^{\prime}+B_{\infty}^{\prime}+A_{f}+B_{f}$. Thus, we are allowed to throw away $A_{\infty}^{\prime}+B_{\infty}^{\prime}$, if an adequate rule is prescribed to retain $A_{f}+B_{f}$. If only $A$ is calculated and it can already yield a quite good approximation, clearly $A_{r}$ and $A_{f}$ must be taken into account except when they are very small.

Redmond [11] suggested that one may use the spectral representation to eliminate the ghost poles. Here we would like to point out that there is a simple and direct way to derive a ghost-free representation for the Dyson solution by the spectral representation. From Eqs. (5), (14) and

$$
G(k)=-D^{-1}\left[\gamma_{\mu} k_{\mu}(1+a)+i M_{t}(1+b)\right]=Z^{-1} \widetilde{G}(k),
$$

where $D$ is given by Eq. (20b), we easily find

$$
\begin{gathered}
1+a=F\left(k^{2}\right)^{-1} F_{\alpha}\left(k^{2}\right) ; \quad 1+b=F\left(k^{2}\right)^{-1} F_{\beta}\left(k^{2}\right), \\
F\left(k^{2}\right)=k^{2} F_{\alpha}\left(k^{2}\right)^{2}+M_{t}^{2} F_{\beta}\left(k^{2}\right)^{2}
\end{gathered}
$$

with $F_{\gamma}\left(k^{2}\right)=\int_{0}^{\infty} d \sigma^{2} f_{\gamma}\left(-\sigma^{2}\right)\left(k^{2}+\sigma^{2}-i \epsilon\right)^{-1}(\gamma=\alpha$ or $\beta)$. It is seen that if $\alpha\left(k^{2}\right)$ and $\beta\left(k^{2}\right)$ are known, so are $F_{\alpha}\left(k^{2}\right)$ and $F_{\beta}\left(k^{2}\right)$. Let us use a subscript $R$ to indicate the results obtained from Eq. (26). Since $a_{R}$ and $b_{R}$ are direct consequences of the spectral representation $\widetilde{G}(k), G_{R}(k)$ deduced from these $a_{R}$ and $b_{R}$ by means of Eq. (5) satisfies Eq.(25) rigorously and is thus ghost-free, because $\widetilde{G}(k)$ is well behaved and possesses the Herglotz property $[12]$. Further one observes that $G_{R}(k)$ obviously satisfies the following Dyson equation:

$$
G_{R}(k)=G^{0}(k)+G^{0}(k) \Sigma_{R}(k) G_{R}(k),
$$


with $G^{0}(k)=-\left[\gamma_{\mu} k_{\mu}-i M_{t}\right]^{-1}$ and $\Sigma_{R}(k)=\gamma_{\mu} k_{\mu} a_{R}\left(k^{2}\right)-i M_{t} b_{R}\left(k^{2}\right)$. Let us insert $\alpha$ and $\beta$ found from Eq.(20) with $a=a_{\delta}+a_{\Delta}$ and $b=b_{\delta}+b_{\Delta}$ calculated according to Eqs.(19) and (21) in $F_{\alpha}$ and $F_{\beta}$. From Eq. (26) we obtain $a_{R}$ and $b_{R}$. We note that the set $\left(a_{R}, b_{R}\right)$ is different from $(a, b)$, though the former is deduced from the latter. In fact, if we substitute $(a, b)$ into Eq. (25), the latter no longer holds, because $G(k)$ found in this way possesses ghost poles, which are $-100.4468 \pm i 55.2553 \mathrm{fm}^{-2}$. The reason is clear. To calculate $a$ and $b$ we need renormalization, which, however, does not ensure that Eq. (25) holds. In Fig. 3 we have compared $\left(a_{R}, b_{R}\right)$ with $(a, b)$. They have different asymptotic behavior and differ widely, though the same $\alpha$ and $\beta$ can be derived from them by Eq. (20). Our numerical result for $\rho_{v}\left(k^{2}\right)$ is well behaved and is qualitatively similar to $\rho_{v}\left(k^{2}\right)$ found for the $\sigma-\omega$ model in [3]. Using Eqs. $(13,17)$ and the relation $\Delta_{v}\left(k^{2}\right)=Y_{v}^{-1} \widetilde{\Delta}_{v}\left(k^{2}\right)$, we can also derive an analytic expression for $\Pi_{v}\left(k^{2}\right)$, which will yield a ghost-free $\Delta_{v}\left(k^{2}\right)$ by means of Eq. (13). However, for lack of spacing both $\rho_{v}$ and $\Pi_{v}$ will not be discussed here. As shown in Refs. [1, 3, 4, 7], if in addition to the $\omega$-meson, other mesons, for instance $\pi, \sigma$ and chiral $\pi-\sigma$ (linear $\sigma$-model), are considered, the baryon spectral functions in the self-consistent HF approximation are regular, even though the $k_{\mu} k_{\nu}$-terms in Eq. (12) are neglected. The question what their effects are in these more complicated cases is being studied.

The work is supported in part by the National Natural Science Foundation of China and the Foundation of Chinese Education Ministry. 


\section{References}

[1] G. Krein, M. Nielsen, R. D. Puff and L. Wilets, Phys. Rev. C 47 (1993) 2485.

[2] C. J. Horowitz and B. D. Serot, Nucl. Phys. A 399 (1983) 529.

[3] S. S. Wu, H. X. Zhang and Y. J. Yao, nucl-th/0011018.

[4] W. D. Brown, R. D. Puff and L. Wilets, Phys. Rev. C 2 (1970) 331; C. A. da Rocha, G. Krein and L. Wilets, Nucl. Phys. A 616 (1997) 625.

[5] A. F. Bielajew and B. D. Serot, Ann. Phys. 156 (1984) 215; A. F. Bielajew, Nucl. Phys. A 404 (1983) 428.

[6] S. S. Wu, J. M. Zhu, K. Z. Liu and Y. J. Yao, Eur. Phys. J. A 6 (1999) 345; Y. J. Yao, H. X. Zhang, Z. M. Zhu, K. Z. Liu and S. S. Wu, Chin. Phys. Lett. 17 (2000) 720.

[7] M. E. Bracco, A. Eiras, G. Krein and L. Wilets, Phys. Rev. C 49 (1994) 1299.

[8] N. M. Kroll, T. D. Lee and B. Zumino, Phys. Rev. 157 (1967) 1376.

[9] D. Lurie, Particles and fields (Interscience, New York, 1968).

[10] J. D. Bjorken and S. D. Drell, Relativistic Quantum Fields (McGrawHill, New York, 1965).

[11] P. J. Redmond, Phys. Rev. 112 (1958) 1404.

[12] L. Wilets, in Meson in Nuclei, ed. M. Rho and D. Wilkinson (NorthHolland, 1979).

Fig. 1 on $\mathrm{P}_{2}$, Fig. 2 on $\mathrm{P}_{6}$, Fig. 3 on $\mathrm{P}_{7}$. 


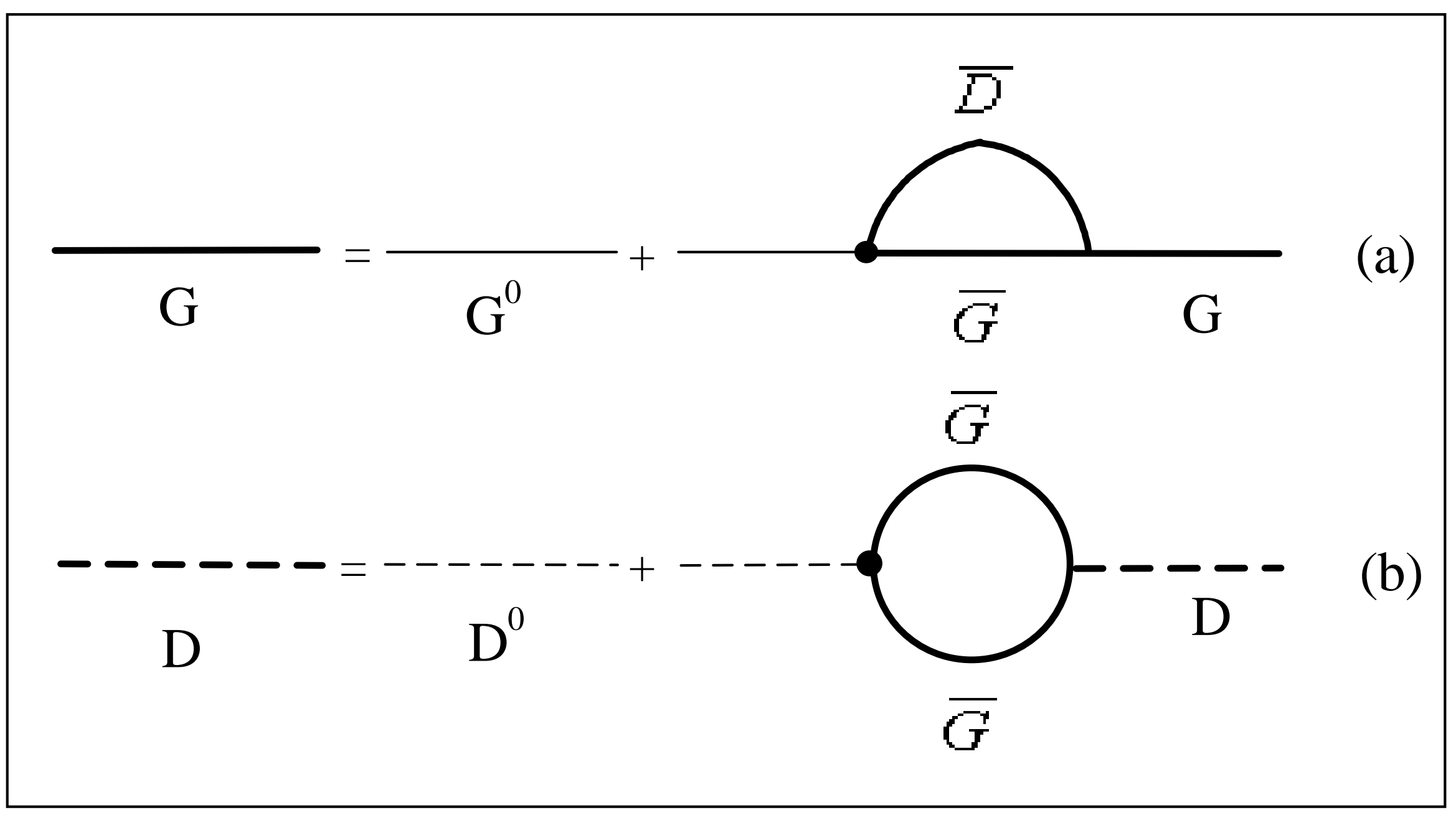

Fig. 1: Diagrammatic representation of the dressed HF approximation. (a) the baryon propagator; (b) the $\omega$-meson propagator. 


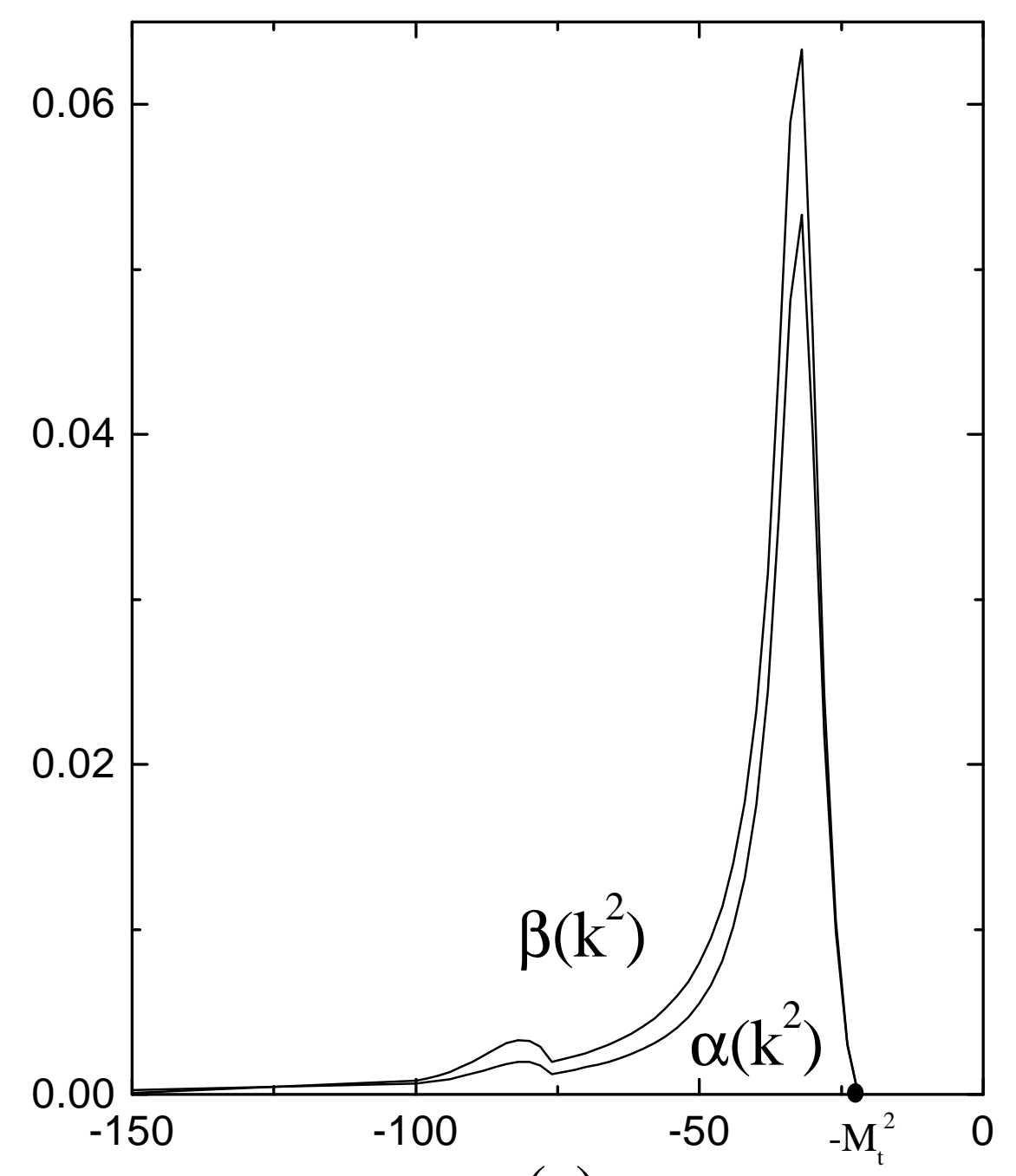

(a)

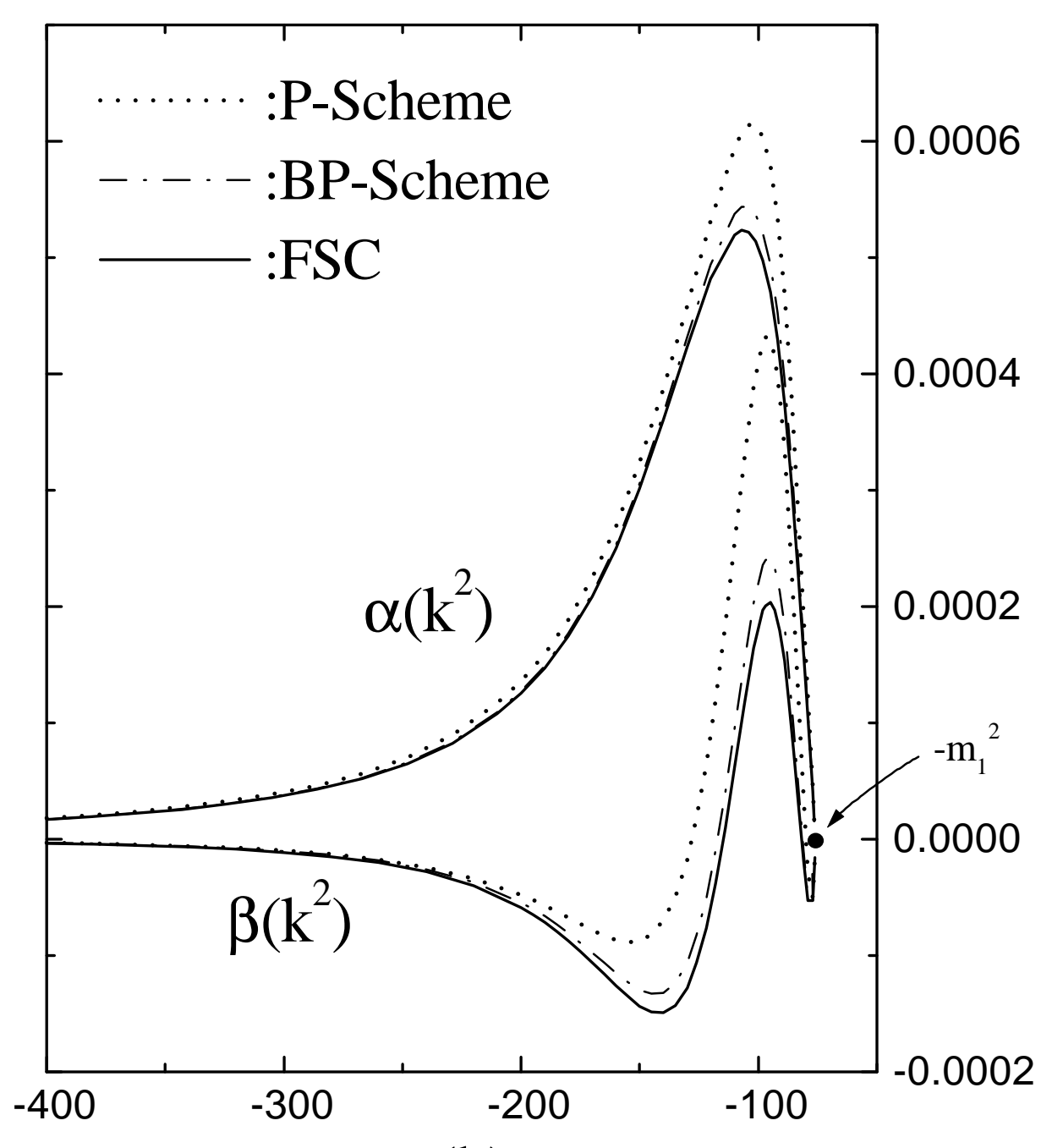

(b)

Fig. 2: The baryon spectral functions $\alpha\left(\mathrm{k}^{2}\right)$ and $\beta\left(\mathrm{k}^{2}\right)$.

(a) with wrong threshold; (b) the correct final result. 


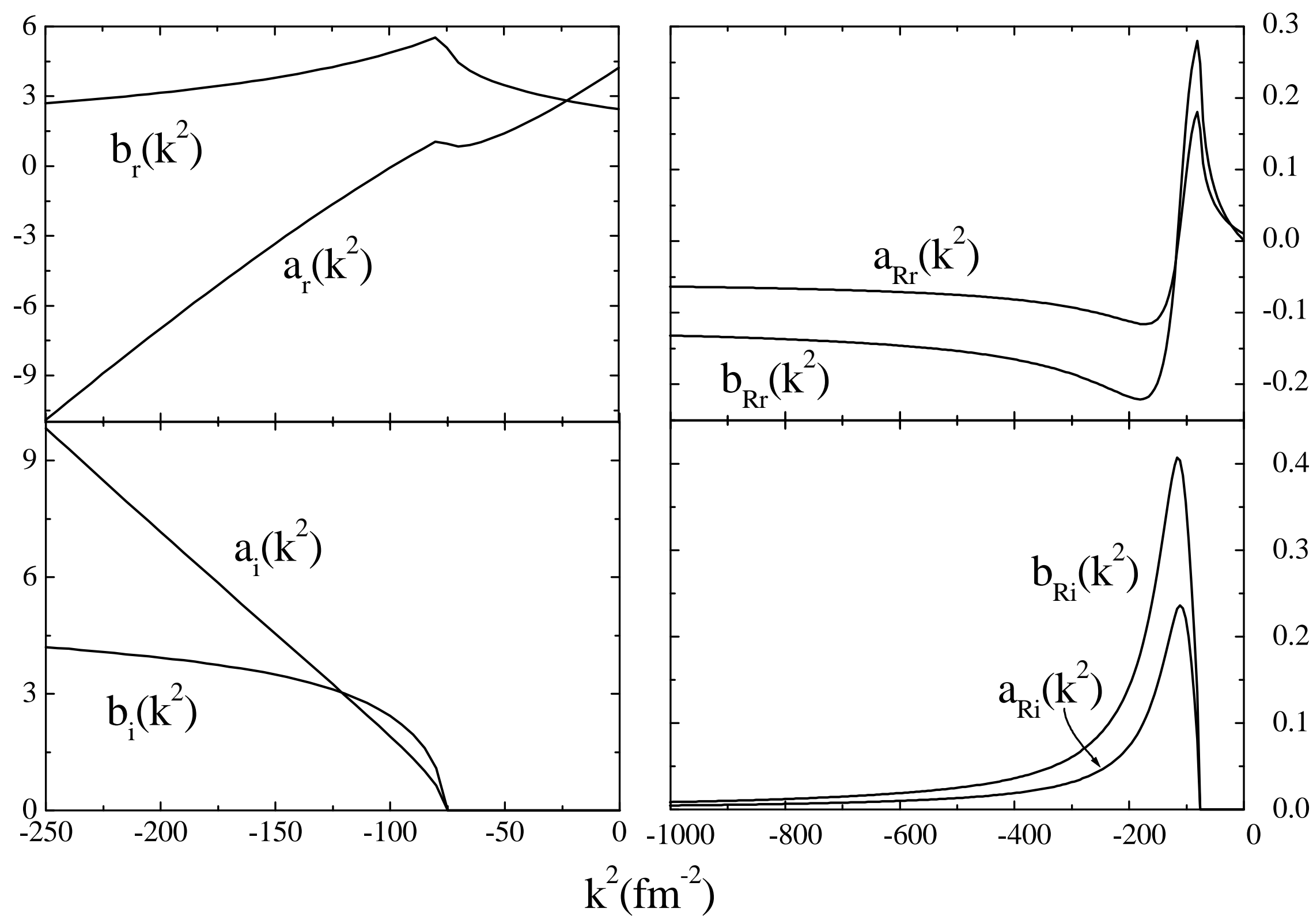

Fig. 3: Comparison betwwen $\left(a\left(k^{2}\right), b\left(k^{2}\right)\right.$ and $\left(a_{R}\left(k^{2}\right), b_{R}\left(k^{2}\right)\right)$.

The subscript ' $r$ ' for the real part, while the ' $i$ ' for the imaginary part. 